\title{
Applications for Certain Classes of Spirallike Functions Defined by the Srivastava-Attiya Operator
}

\section{Jae Ho Choi}

Department of Mathematics Education, Daegu National University of Education, Daegu, Korea

Email: choijh@dnue.ac.kr

How to cite this paper: Choi, J.H. (2018) Applications for Certain Classes of Spirallike Functions Defined by the Srivastava-Attiya Operator. Advances in Pure Mathematics, 8, 615-623.

https://doi.org/10.4236/apm.2018.86035

Received: May 17, 2018

Accepted: June 26, 2018

Published: June 29, 2018

Copyright $\odot 2018$ by author and Scientific Research Publishing Inc. This work is licensed under the Creative Commons Attribution International License (CC BY 4.0).

http://creativecommons.org/licenses/by/4.0/

\begin{abstract}
Let the function $f$ be analytic in $\mathbb{U}=\{z: z \in \mathbb{C}$ and $|z|<1\}$ and be given by $f(z)=z+\sum_{k=2}^{\infty} a_{k} z^{k}$. In this paper, making use of the Srivastava-Attiya operator $\mathcal{L}_{s, b}$, we introduce two classes of analytic functions and investigate some convolution properties and coefficient estimates for these classes. Furthermore, several inclusion properties involving these and other families of integral operators are also considered.
\end{abstract}

\section{Keywords}

Analytic Function, Hadamard Product, Subordination, Srivastava-Attiya Operator

\section{Introduction and Definitions}

Let $\mathcal{A}$ denote the class of functions $f(z)$ of the form

$$
f(z)=z+\sum_{k=2}^{\infty} a_{k} z^{k}
$$

which are analytic in the open unit disk $\mathbb{U}=\{z: z \in \mathbb{C}$ and $|z|<1\}$. Also let $f$ and $g$ be analytic in $\mathbb{U}$ with $f(0)=g(0)$. Then we say that $f$ is subordinate to $g$ in $\mathbb{U}$, written $f \prec g$ or $f(z) \prec g(z)$, if there exists the Schwarz function $w$, analytic in $\mathbb{U}$ such that $w(0)=0,|w(z)|<1$ and $f(z)=g(w(z)) \quad(z \in \mathbb{U})$. We also observe that

$$
f(z) \prec g(z) \quad \text { in } \mathbb{U}
$$

if and only if

$$
f(0)=g(0) \text { and } f(\mathbb{U}) \subset g(\mathbb{U})
$$


whenever $g$ is univalent in $\mathbb{U}$.

For functions $f_{j}(z) \in \mathcal{A}$, given by

$$
f_{j}(z)=z+\sum_{k=2}^{\infty} a_{k, j} z^{k} \quad(j=1,2),
$$

we define the Hadamard product (or convolution) of $f_{1}(z)$ and $f_{2}(z)$ by

$$
\left(f_{1} * f_{2}\right)(z)=z+\sum_{k=2}^{\infty} a_{k, 1} a_{k, 2} z^{k}=\left(f_{2} * f_{1}\right)(z) \quad(z \in \mathbb{U}) .
$$

Making use of the principle of subordination between analytic functions, Bhoosnurmath and Devadas [1] considered the subclasses $\mathcal{S}^{\alpha}[A, B]$ and $\mathcal{K}^{\alpha}[A, B]$ of the class $\mathcal{A}$ for $|\alpha|<\frac{\pi}{2}$ and $-1 \leq B<A \leq 1$ as following (see also [2] and [3]):

$$
\mathcal{S}^{\alpha}[A, B]=\left\{f \in \mathcal{A}: \mathrm{e}^{i \alpha} \frac{z f^{\prime}(z)}{f(z)} \prec \cos \alpha\left(\frac{1+A z}{1+B z}\right)+i \sin \alpha(z \in \mathbb{U})\right\},
$$

and

$$
\mathcal{K}^{\alpha}[A, B]=\left\{f \in \mathcal{A}: \mathrm{e}^{i \alpha} \frac{\left(z f^{\prime}(z)\right)^{\prime}}{f(z)} \prec \cos \alpha\left(\frac{1+A z}{1+B z}\right)+i \sin \alpha(z \in \mathbb{U})\right\} .
$$

We note that

$$
\mathcal{S}^{0}[A, B]=\mathcal{S}[A, B], \quad \mathcal{K}^{0}[A, B]=\mathcal{K}[A, B] \quad(-1 \leq B<A \leq 1),
$$

where the classes $\mathcal{S}[A, B]$ and $\mathcal{K}[A, B]$ are introduced and studied by many authors (see [4] [5] and [6]). Furthermore, $\mathcal{S}^{\alpha}[1,-1] \equiv \mathcal{S}(\alpha)$ denote the $\alpha$-spirallike functions studied by Spacek [7], which are univalent in $\mathbb{U}$.

With a view to define the Srivastava-Attiya transform, we recall here a general Hurwitz-Lerch zeta function, which is defined in [8] by the following series:

$$
\begin{gathered}
\Phi(z, s, a):=\frac{1}{a^{s}}+\sum_{k=1}^{\infty} \frac{z^{k}}{(k+a)^{s}} \\
\left(a \in \mathbb{C} \backslash \mathbb{Z}_{0}^{-}=\{0,-1,-2, \cdots\} ; s \in \mathbb{C} \text { when } z \in \mathcal{U} ; \operatorname{Re}(s)>1 \text { when }|z|=1\right)
\end{gathered}
$$

For further interesting properties and characteristics of the Hurwitz-Lerch Zeta and other related functions $\Phi(z, s, a)$ see [9] [10] and [11].

Recently, Srivastava and Attiya [12] have introduced the linear operator $\mathcal{L}_{s, b}: \mathcal{A} \rightarrow \mathcal{A}$, defined in terms of the Hadamard product by

$$
\mathcal{L}_{s, b}(f)(z)=\mathcal{G}_{s, b}(z) * f(z) \quad\left(z \in \mathbb{U} ; b \in \mathbb{C} \backslash \mathbb{Z}_{0}^{-} ; s \in \mathbb{C}\right),
$$

where

$$
\mathcal{G}_{s, b}=(1+b)^{s}\left[\Phi(z, s, b)-b^{-s}\right] \quad(z \in \mathbb{U}) .
$$

The operator $\mathcal{L}_{s, b}$ is now popularly known in the literature as the Srivastava-Attiya operator. Various class-mapping properties of the operator $\mathcal{L}_{s, b}$ (and its variants) are discussed in the recent works of Srivastava and Attiya 
[12], Liu [13], Murugusundaramoorthy [14], Yuan and Liu [15] and others.

It is easy to observe from (1.1) and (1.4) that

$$
\mathcal{L}_{s, b}(f)(z)=z+\sum_{k=2}^{\infty}\left(\frac{1+b}{k+b}\right)^{s} a_{k} z^{k}
$$

We note that:

1) $\mathcal{L}_{0, b}(f)(z)=f(z)$;

2) $\mathcal{L}_{1,0}(f)(z)=\mathcal{L}(f)(z)=\int_{0}^{z} \frac{f(t)}{t} \mathrm{~d} t \quad(f \in \mathcal{A}) \quad$ (see Alexander [16]);

3) $\mathcal{L}_{m, 1}(f)(z)=\mathcal{I}^{m} f(z) \quad\left(m \in \mathbb{N}_{0}=\mathbb{N} \bigcup\{0\}=\{0,1,2,3, \cdots\}\right) \quad$ (see Flett [17]);

4) $\mathcal{L}_{\gamma, 1}(f)(z)=\mathcal{Q}^{\gamma} f(z) \quad(\gamma>0) \quad$ (see Jung et al. [18]);

5) $\mathcal{L}_{m, 0}(f)(z)=\mathcal{L}^{m} f(z) \quad\left(m \in \mathbb{N}_{0}\right) \quad$ (see Sălăgean [19]).

It is easily verified from (1.6) that

$$
\begin{gathered}
z\left(\mathcal{L}_{s, b}(f)(z)\right)^{\prime}=(b+1) \mathcal{L}_{s-1, b}(f)(z)-b \mathcal{L}_{s, b}(f)(z) \\
\left(f \in \mathcal{A} ; b \in \mathbb{C} \backslash \mathbb{Z}_{0}^{-} ; s \in \mathbb{C}\right)
\end{gathered}
$$

Next, by using the linear operator $\mathcal{L}_{s, b}$, we introduce the following new classes of analytic functions for $b \in \mathbb{C} \backslash \mathbb{Z}_{0}^{-}, s \in \mathbb{C},|\alpha|<\frac{\pi}{2}$ and $-1 \leq B<A \leq 1:$

$$
\mathcal{S}_{s, b}^{\alpha}[A, B]:=\left\{f \in \mathcal{A}: \mathcal{L}_{s, b}(f)(z) \in \mathcal{S}^{\alpha}[A, B](z \in \mathbb{U})\right\}
$$

and

$$
\mathcal{K}_{s, b}^{\alpha}[A, B]:=\left\{f \in \mathcal{A}: \mathcal{L}_{s, b}(f)(z) \in \mathcal{K}^{\alpha}[A, B](z \in \mathbb{U})\right\} .
$$

It follows from the definitions (1.8) and (1.9) that

$$
f(z) \in \mathcal{K}_{s, b}^{\alpha}[A, B] \Leftrightarrow z f^{\prime}(z) \in \mathcal{S}_{s, b}^{\alpha}[A, B] \quad(z \in \mathbb{U}) .
$$

In this article, we investigate some convolution properties and coefficient estimates for the classes $\mathcal{S}_{s, b}^{\alpha}[A, B]$ and $\mathcal{K}_{s, b}^{\alpha}[A, B]$. Furthermore, several inclusion properties and relevant connections of the results presented here with those obtained in earlier works are also discussed.

\section{Convolution Properties and Coefficient Estimates}

Unless otherwise mentioned, we will assume in the reminder of this paper that $-1 \leq B<A \leq 1,|\alpha|<\frac{\pi}{2}$ and $|\zeta|=1$. In order to establish our convolution properties, we shall need the following lemmas due to Bhoosnurnath and Devadas [1] [2].

Lemma 2.1 ([1]). The function $f(z)$ defined by (1.1) is in the class $\mathcal{S}^{\alpha}[A, B]$ if and only if

$$
\frac{1}{z}\left\{f(z) *(1-M z) \frac{z}{(1-z)^{2}}\right\} \neq 0 \quad(z \in \mathbb{U}),
$$


where

$$
M=\frac{\mathrm{e}^{i \alpha}+(A \cos \alpha+i B \sin \alpha) \zeta}{(A-B) \zeta \cos \alpha} .
$$

Lemma 2.2 ([2] Lemma 3 with $n=1$ ). The function $f(z)$ defined by (1.1) is in the class $\mathcal{K}^{\alpha}[A, B]$ if and only if

$$
\frac{1}{z}\left\{f(z) *(1-N z) \frac{z}{(1-z)^{3}}\right\} \neq 0 \quad(z \in \mathbb{U}),
$$

where

$$
N=\frac{2 \mathrm{e}^{i \alpha}+[(A+B) \cos \alpha+i 2 B \sin \alpha] \zeta}{(A-B) \zeta \cos \alpha} .
$$

We begin by proving the following theorem.

Theorem 2.3 The function $f(z)$ defined by (1.1) is in the class $\mathcal{K}_{s, b}^{\alpha}[A, B]$ if and only if

$$
1-\sum_{k=2}^{\infty} \frac{(k-1+k B \zeta) \mathrm{e}^{i \alpha}-(A \cos \alpha+i B \sin \alpha) \zeta}{(A-B) \zeta \cos \alpha}\left(\frac{1+b}{k+b}\right)^{s} a_{k} z^{k-1} \neq 0 \quad(z \in \mathbb{U}) .
$$

Proof. From Lemma 2.1, we find that $f(z) \in \mathcal{S}_{s, b}^{\alpha}[A, B]$ if and only if

$$
\frac{1}{z}\left[\mathcal{L}_{s, b}(f)(z) *(1-M z) \frac{z}{(1-z)^{2}}\right] \neq 0 \quad(z \in \mathbb{U}),
$$

where $M$ is given by (2.2). Then, by applying (1.6), the left hand side of (2.5) becomes

$$
\begin{aligned}
& \frac{1}{z}\left[\mathcal{L}_{s, b}(f)(z) *\left(\frac{z}{(1-z)^{2}}-\frac{M z}{(1-z)^{2}}\right)\right] \\
& =\frac{1}{z}\left[z\left(\mathcal{L}_{s, b}(f)(z)\right)^{\prime}-M\left\{z\left(\mathcal{L}_{s, b}(f)(z)\right)^{\prime}-\mathcal{L}_{s, b}(f)(z)\right\}\right] \\
& =1-\sum_{k=2}^{\infty} \frac{(k-1+k B \zeta) \mathrm{e}^{i \alpha}-(A \cos \alpha+i B \sin \alpha) \zeta}{(A-B) \zeta \cos \alpha}\left(\frac{1+b}{k+b}\right)^{s} a_{k} z^{k-1},
\end{aligned}
$$

which completes the proof of Theorem 2.3.

Theorem 2.4 The function $f(z)$ defined by $(1.1)$ is in the class $\mathcal{K}_{s, b}^{\alpha}[A, B]$ if and only if

$$
1-\sum_{k=2}^{\infty} k \frac{(k-1) \mathrm{e}^{i \alpha}-[(A-k B) \cos \alpha-i(k-1) B \sin \alpha] \zeta}{(A-B) \zeta \cos \alpha}\left(\frac{1+b}{k+b}\right)^{s} a_{k} z^{k-1} \neq 0(z \in \mathbb{U}) .
$$

Proof. From Lemma 2.2, we observe that $f(z) \in \mathcal{K}_{s, b}^{\alpha}[A, B]$ if and only if

$$
\frac{1}{z}\left[\mathcal{L}_{s, b}(f)(z) *(1-N z) \frac{z}{(1-z)^{3}}\right] \neq 0 \quad(z \in \mathbb{U}),
$$

where $N$ is given by (2.4). Then, by using (1.6), the left hand side of (2.6) may be written as 


$$
\begin{aligned}
& \frac{1}{z}\left[\mathcal{L}_{s, b}(f)(z) *\left(\frac{z}{(1-z)^{3}}-\frac{N z^{2}}{(1-z)^{3}}\right)\right] \\
& =\frac{1}{z}\left[\frac{1}{2} z\left(z \mathcal{L}_{s, b}(f)(z)\right)^{\prime \prime}-N\left\{\frac{1}{2} z\left(z \mathcal{L}_{s, b}(f)(z)\right)^{\prime \prime}-z\left(\mathcal{L}_{s, b}(f)(z)\right)^{\prime}\right\}\right] \\
& =1-\sum_{k=2}^{\infty} k \frac{(k-1) \mathrm{e}^{i \alpha}-[(A-k B) \cos \alpha-i(k-1) B \sin \alpha] \zeta}{(A-B) \zeta \cos \alpha}\left(\frac{1+b}{k+b}\right)^{s} a_{k} z^{k-1},
\end{aligned}
$$

which evidently proves Theorem 2.4 .

Next, we determine coefficients estimates for a function of the form (1.1) to be in the classes $\mathcal{S}_{s, b}^{\alpha}[A, B]$ and $\mathcal{K}_{s, b}^{\alpha}[A, B]$.

Theorem 2.5 Let $b>-1$ and $s \geq 0$. The function $f(z)$ defined by (1.1) is in the class $\mathcal{S}_{s, b}^{\alpha}[A, B]$ if its coefficients satisfy the condition

$$
\sum_{k=2}^{\infty}\left(k-1+\left|A \cos \alpha+i B \sin \alpha-k B \mathrm{e}^{i \alpha}\right|\right)\left(\frac{1+b}{k+b}\right)^{s}\left|a_{k}\right| \leq(A-B) \cos \alpha .
$$

Proof. Since

$$
\begin{aligned}
& \left|1-\sum_{k=2}^{\infty} \frac{(k-1+k B \zeta) \mathrm{e}^{i \alpha}-(A \cos \alpha+i B \sin \alpha) \zeta}{(A-B) \zeta \cos \alpha}\left(\frac{1+b}{k+b}\right)^{s} a_{k} z^{k-1}\right| \\
& \geq 1-\sum_{k=2}^{\infty}\left|\frac{(k-1+k B \zeta) \mathrm{e}^{i \alpha}-(A \cos \alpha+i B \sin \alpha) \zeta}{(A-B) \zeta \cos \alpha}\right|\left(\frac{1+b}{k+b}\right)^{s}\left|a_{k}\right|,
\end{aligned}
$$

and

$$
\begin{aligned}
& \left|\frac{(k-1+k B \zeta) \mathrm{e}^{i \alpha}-(A \cos \alpha+i B \sin \alpha) \zeta}{(A-B) \zeta \cos \alpha}\right| \\
& =\frac{\left|(k-1) \mathrm{e}^{i \alpha}-\left(A \cos \alpha+i B \sin \alpha-k B \mathrm{e}^{i \alpha}\right) \zeta\right|}{(A-B) \cos \alpha} \\
& \leq \frac{(k-1)+\left|A \cos \alpha+i B \sin \alpha-k B \mathrm{e}^{i \alpha}\right|}{(A-B) \cos \alpha},
\end{aligned}
$$

by virtue of Theorem 2.3, we conclude that $f(z) \in \mathcal{S}_{s, b}^{\alpha}[A, B]$. Thus, the proof of Theorem 2.5 is completed.

By using arguments similar to those above with Theorem 2.4, we can prove the following theorem.

Theorem 2.6 Let $b>-1$ and $s \geq 0$. The function $f(z)$ defined by (1.1) is in the class $\mathcal{K}_{s, b}^{\alpha}[A, B]$ if its coefficients satisfy the condition

$$
\sum_{k=2}^{\infty} k\{k-1+|(A-k B) \cos \alpha-i(k-1) B \sin \alpha|\}\left(\frac{1+b}{k+b}\right)^{s}\left|a_{k}\right| \leq(A-B) \cos \alpha .
$$

\section{Inclusion Properties and Applications}

To prove the inclusion properties for the classes $\mathcal{S}_{s, b}^{\alpha}[A, B]$ and $\mathcal{K}_{s, b}^{\alpha}[A, B]$, we shall require the following lemma due to Eenigenburg et al. [20].

Lemma 3.1 ([20]). Let $h(z)$ be convex univalent in $\mathbb{U}$ with 
$\operatorname{Re}\{\beta h(z)+v\}>0$ for all $z \in \mathbb{U}$. If $p(z)$ is analytic in $\mathbb{U}$ with $p(0)=h(0)$, then

$$
p(z)+\frac{z p^{\prime}(z)}{\beta p(z)+v} \prec h(z) \quad(z \in \mathbb{U})
$$

implies that $p(z) \prec h(z) \quad(z \in \mathbb{U})$.

By applying Lemma 3.1, we prove

Theorem 3.2 Let $b>-1$ and $s \geq 0$. If

$$
\operatorname{Re}\left\{\mathrm{e}^{-i \alpha} \frac{z}{1+B z}\right\}>-\frac{b+1}{(A-B) \cos \alpha} \quad(z \in \mathbb{U}),
$$

then

$$
\mathcal{S}_{s-1, b}^{\alpha}[A, B] \subset \mathcal{S}_{s, b}^{\alpha}[A, B] .
$$

Proof. Let $f(z) \in \mathcal{S}_{s-1, b}^{\alpha}[A, B]$ for $b>-1$ and $s \geq 0$, and set

$$
p(z)=\mathrm{e}^{i \alpha} \frac{z\left(\mathcal{L}_{s, b}(f)(z)\right)^{\prime}}{\mathcal{L}_{s, b}(f)(z)} \quad(z \in \mathbb{U}),
$$

where $p(z)$ is analytic in $\mathbb{U}$ with $p(0)=\mathrm{e}^{i \alpha}$. By applying the identity (1.7), we obtain

$$
\mathrm{e}^{-i \alpha} p(z)+b=(b+1) \frac{\mathcal{L}_{s-1, b}(f)(z)}{\mathcal{L}_{s, b}(f)(z)} .
$$

Making use of the logarithmic differentiation on both side in (3.3), we have

$$
p(z)+\frac{z p^{\prime}(z)}{\mathrm{e}^{-i \alpha} p(z)+b} \prec \cos \alpha\left(\frac{1+A z}{1+B z}\right)+i \sin \alpha=h(z) .
$$

Since the function $h(z)$ is convex univalent in $\mathbb{U}$ with $h(0)=\mathrm{e}^{i \alpha}$, from (3.1) we see that

$$
\operatorname{Re}\left\{\mathrm{e}^{-i \alpha} h(z)+b\right\}>0 \quad(z \in \mathbb{U}) .
$$

Thus, by using Lemma 3.1 and (3.4), we observe that $p(z) \prec h(z)$ in $\mathbb{U}$, so that $f(z) \in \mathcal{S}_{s, b}^{\alpha}[A, B]$. This completes the proof of theorem 3.2.

Theorem 3.3 Let $b>-1$ and $s \geq 0$. Suppose that (3.1) holds for all $z \in \mathbb{U}$. Then

$$
\mathcal{K}_{s-1, b}^{\alpha}[A, B] \subset \mathcal{K}_{s, b}^{\alpha}[A, B] .
$$

Proof. Applying (1.10) and Theorem 3.2, we observe that

$$
\begin{gathered}
f(z) \in \mathcal{K}_{s-1, b}^{\alpha}[A, B] \Leftrightarrow z f^{\prime}(z) \in \mathcal{S}_{s-1, b}[A, B] \\
\Rightarrow z f^{\prime}(z) \in \mathcal{S}_{s, b}^{\alpha}[A, B] \\
\Leftrightarrow f(z) \in \mathcal{K}_{s, b}^{\alpha}[A, B],
\end{gathered}
$$

which evidently proves Theorem 3.3 .

Putting $b=1$ and $A=-B=1$ in Theorem 3.2 and 3.3 , we have the following corollary. 
Corollary 3.4 Suppose that $s \geq 0$ and

$$
\operatorname{Re}\left\{\mathrm{e}^{-i \alpha} \frac{z}{1-z}\right\}>-\frac{1}{\cos \alpha} \quad(z \in \mathbb{U}) .
$$

Then

$$
\mathcal{S}_{s-1,1}^{\alpha}[1,-1] \subset \mathcal{S}_{s, 1}^{\alpha}[1,-1]
$$

and

$$
\mathcal{K}_{s-1,1}^{\alpha}[1,-1] \subset \mathcal{K}_{s, 1}^{\alpha}[1,-1] .
$$

Finally, we consider the generalized Bernardi-Libera-Livingston integral operator $\mathcal{J}_{\sigma}(f)$ defined by (cf. [21] [22] and [23])

$$
\mathcal{J}_{\sigma}(f) \equiv \mathcal{J}_{\sigma}(f)(z):=\frac{\sigma+1}{z^{\sigma}} \int_{0}^{z} t^{\sigma-1} f(t) \mathrm{d} t \quad(f \in \mathcal{A} ; \sigma>-1) .
$$

Theorem 3.5 Let $b>-1, s \geq 0$ and $\sigma>-1$. Suppose that

$$
\operatorname{Re}\left\{\mathrm{e}^{-i \alpha} \frac{z}{1+B z}\right\}>-\frac{\sigma+1}{(A-B) \cos \alpha} \quad(z \in \mathbb{U}) \text {. }
$$

If $f(z) \in \mathcal{S}_{s, b}^{\alpha}[A, B]$, then $\mathcal{J}_{\sigma}(f)(z) \in \mathcal{S}_{s, b}^{\alpha}[A, B]$.

Proof. If we set

$$
p(z)=\mathrm{e}^{i \alpha} \frac{z\left(\mathcal{L}_{s, b} \mathcal{J}_{\sigma}(f)(z)\right)^{\prime}}{\mathcal{L}_{s, b} \mathcal{J}_{\sigma}(f)(z)} \quad(z \in \mathbb{U}),
$$

where $p(z)$ is analytic in $\mathbb{U}$ with $p(0)=\mathrm{e}^{i \alpha}$. By virtue of (3.5), we observe that

$$
z\left(\mathcal{L}_{s, b} \mathcal{J}_{\sigma}(f)(z)\right)^{\prime}=(\sigma+1) \mathcal{L}_{s, b}(f)(z)-\sigma \mathcal{L}_{s, b} \mathcal{J}_{\sigma}(f)(z) \quad(z \in \mathbb{U})
$$

In view of (3.7) and (3.8), we have

$$
\mathrm{e}^{-i \alpha} p(z)+\sigma=(\sigma+1) \frac{\mathcal{L}_{s, b}(f)(z)}{\mathcal{L}_{s, b} \mathcal{J}_{\sigma}(f)(z)} .
$$

By using same argument as in the proof of Theorem 3.2 with (3.6), we conclude that $\mathcal{J}_{\sigma}(f)(z) \in \mathcal{S}_{s, b}^{\alpha}[A, B]$. This evidently completes the proof of Theorem 3.5.

Theorem 3.6 Let $b>-1, s \geq 0$ and $\sigma>-1$. Suppose that (3.6) holds for all $z \in \mathbb{U}$. If $f(z) \in \mathcal{K}_{s, b}^{\alpha}[A, B]$, then $\mathcal{J}_{\sigma}(f)(z) \in \mathcal{K}_{s, b}^{\alpha}[A, B]$.

Proof. By using Theorem 3.4, it follows that

$$
\begin{aligned}
f(z) & \in \mathcal{K}_{s, b}[A, B] \Leftrightarrow z f^{\prime}(z) \in \mathcal{S}_{s, b}[A, B] \\
& \Rightarrow \mathcal{J}_{\sigma}\left(z f^{\prime}(z)\right) \in \mathcal{S}_{s, b}[A, B] \\
\Leftrightarrow & z\left(\mathcal{J}_{\sigma}(f)(z)\right)^{\prime} \in \mathcal{S}_{s, b}[A, B] \\
& \Rightarrow \mathcal{J}_{\sigma}(f)(z) \in \mathcal{K}_{s, b}[A, B]
\end{aligned}
$$

which completes the proof of Theorem 3.6. 


\section{Acknowledgements}

This work was supported by Daegu National University of Education Research grant in 2017.

\section{References}

[1] Bhoosnurmath, S.S. and Devadas, M.V. (1996) Subclasses of Spirallike Functions Defined by Subordination. Journal of Analysis Madras, 4, 173-183.

[2] Bhoosnurmath, S.S. and Devadas, M.V. (1997) Subclasses of Spirallike Functions Defined by Ruschweyh Derivatives. Tamkang Journal of Mathematics, 28, 59-65.

[3] Nikitin, S.V. (1987) A Class of Regular Functions, Current Problem in Function Theory (in Russian). Teberda 1985, Rostov Gos. Univ., Rostov-on-Don, 143-147.

[4] Ahuja, O.P. (1993) Families of Analytic Functions Related to Ruscheweyh Derivatives and Subordinate to Convex Functions. Yokohama Mathematical Journal, 41, 39-50.

[5] Goel, R.M. and Mehrok, B.S. (1981) On the Coefficients of a Subclass of Starlike Functions. Indian Journal of Pure and Applied Mathematics, 12, 634-647.

[6] Silverman, H. and Silvia, E.M. (1985) Subclasses of Starlike Functions Subordinate to Convex Functions. Canadian Journal of Mathematics, 1, 48-61. https://doi.org/10.4153/CJM-1985-004-7

[7] Spacek, L. (1932) Contribution à la theorie des fonctions univalents. Casopis pro Pestovani Matematiky Fysiky, 62, 12-19.

[8] Srivastava, H.M. and Choi, J. (2001) Series Associated with the Zeta and Related Function. Kluwer Academic Publishers, Dordrecht.

https://doi.org/10.1007/978-94-015-9672-5

[9] Ferreira, C. and Lopez, J.L. (2004) Asymptotic Expansions of the Hurwitz-Lerch Zeta Function. Journal of Mathematical Analysis and Applications, 298, 210-224. https://doi.org/10.1016/j.jmaa.2004.05.040

[10] Lin, S.D., Srivastava, H.M. and Wang, P.Y. (2006) Some Expansion Formulas for a Class of Generalized Hurwitz-Lerch Zeta Functions. Integral Transforms and Special Functions, 17, 817-827. https://doi.org/10.1080/10652460600926923

[11] Srivastava, H.M., Jankov, D., Pogány, T.K. and Saxena, R.K. (2011) Two-Side Inequalities for the Extended Hurwitz-Lerch Zeta Function. Computers \& Mathematics with Applications, 62, 516-522.

[12] Srivastava, H.M. and Attiya, A.A. (2007) An Integral Operator Associated with the Hurwitz-Lerch Zeta Function and Differential Subordination. Integral Transforms and Special Functions, 18, 207-216. https://doi.org/10.1080/10652460701208577

[13] Liu, J.L. (2011) Sufficient Conditions for Strongly Starlike Functions Involving the Generalized Srivastava-Attiya Operator. Integral Transforms and Special Functions, 22, 79-90. https://doi.org/10.1080/10652469.2010.498110

[14] Murugusundaramoorthy, G. (2012) Subordination Results for Spiral-Like Functions Associated with the Srivastava-Attiya Operator. Integral Transforms and Special Functions, 23, 97-103. https://doi.org/10.1080/10652469.2011.562501

[15] Yuan, S.M. and Liu, Z.M. (2011) Some Properties of Two Subclasses of k-Fold Symmetric Functions Associated with Srivastava-Attiya Operator. Applied Mathematics and Computation, 218, 1136-1141. https://doi.org/10.1016/j.amc.2011.03.080

[16] Alexander, J.W. (1915) Functions Which Map the Interior of the Unit Corcle upon Simple Regions. Annals of Mathematics, 17, 12-22. https://doi.org/10.2307/2007212 
[17] Flett, T.M. (1972) The Dual of an Inequality of Hardy and Littlewood and Some Related Inequalities. Journal of Mathematical Analysis and Applications, 38, 746-765. https://doi.org/10.1016/0022-247X(72)90081-9

[18] Jung, I.B., Kim, Y.C. and Srivastava, H.M. (1993) The Hardy Space of Analytic Functions Associated with Certain One-Parameter Families of Integral Operators. Journal of Mathematical Analysis and Applications, 176, 138-147. https://doi.org/10.1006/jmaa.1993.1204

[19] Salagean, G.S. (1983) Subclasses of Univalent Functions. Lecture Notes in Mathematics, Vol. 1013, Springer, Berlin, 362-372.

[20] Eenigenburg, P., Miller, S.S., Mocanu, P.T. and Reade, M.O. (1983) On a Briot-Bouquet Differential Subordination. In: General Inequalities 3, International Series of Numerical Mathematics, Vol. 64, Birkhäuser Verlag, Basel, 339-348. https://doi.org/10.1007/978-3-0348-6290-5_26

[21] Bernardi, S.D. (1969) Convex and Starlike Univalent Functions. Transactions of the American Mathematical Society, 135, 429-446. https://doi.org/10.1090/S0002-9947-1969-0232920-2

[22] Libera, R.J. (1965) Some Classes of Regular Univalent Functions. Proceedings of the American Mathematical Society, 16, 755-758. https://doi.org/10.1090/S0002-9939-1965-0178131-2

[23] Srivastava, H.M. and Owa, S. (1992) Current Topics in Analytic Function Theory. World Scientific Publishing Company, Singapore, London, and Hong Kong. 